\title{
DIRECT PULPCAPPING WITH A NEW MATERIAL BIODENTINE: A CASE REPORT
}

Nitish Kumar Neelagiri1 ${ }^{1}$ Geeta I. B2 ${ }^{2}$ Shubhashini Narayanaswamy³, Vinay Chandra R ${ }^{4}$ Aniketh T. N5

\section{HOW TO CITE THIS ARTICLE:}

Nitish Kumar Neelagiri, Geeta I. B, Shubhashini Narayanaswamy, Vinay Chandra R, Aniketh T. N. "Direct Pulpcapping with a New Material Biodentine: A Case Report". Journal of Evolution of Medical and Dental Sciences 2015; Vol. 4, Issue 34, April 27; Page: 5990-5994, DOI: 10.14260/jemds/2015/873

ABSTRACT: Biodentine ${ }^{\mathrm{TM}}$ is a new bioactive cement with dentin-like mechanical properties, which can be used as a dentin substitute on crowns and roots, has a positive effect on vital pulp cells and stimulates tertiary dentin formation. This prompted its use for direct pulp capping after iatrogenic pulp exposure at tooth 36 in a 22-years-old male patient. Direct pulp capping was done with Biodentine and a composite filling was placed to replace the dentin and enamel layers. At the followup visit of 6 months the tooth was clinically normal and tested positive for sensitivity and negative for percussion. The IOPA radiograph showed the apical region without any pathological finding. Due to its improved material properties, Biodentine ${ }^{\mathrm{TM}}$ can be an alternative to conventional calcium hydroxide-based materials for direct pulp capping and, may contribute to the long-term maintenance of tooth vitality.

KEYWORDS: Biodentine, direct pulp capping, maintenance of tooth vitality, reparative dentin.

INTRODUCTION: This has been documented by basic research and clinical studies with reported success rates in excess of $80 \%$ for direct pulp capping procedures in humans (Baume and Holz, Hørsted et al. 1985). Calcium hydroxide has been shown to promote the differentiation of odontoblasts or odontoblast-like cells, which will form a hard tissue bridge in the pulp, i.e. calcium hydroxide contributes actively to the formation of new hard tissue by induction and up-regulation of the differentiation of odontoblast-like cells (Schröder 1972) ${ }^{1}$. However, calcium hydroxide has some major drawbacks that have put into question its continued usage. The compound exhibits poor bonding to dentin, mechanical instability and continued resorption after placement. Thus, innovative materials have been employed as pulp capping agents and include hydrophilic resins, resin-modified glass ionomer cements, ozone technology, lasers, resins combined with bioactive agents and mineral trioxide aggregate (MTA). ${ }^{2}$

A new bioactive cement, Biodentine ${ }^{\mathrm{TM}} \mathrm{n}$ (Septodont, St. Maur-des-Fossés, France), was recently launched on the dental market as a dentin substitute. It shares both its indications and mode of action with calcium hydroxide, but does not have its drawbacks. Biodentine ${ }^{\mathrm{TM}}$ consists of a powder in a capsule and liquid in a pipette. The powder mainly contains tricalcium and dicalcium silicate, the principal component of Portland cement, as well as calcium carbonate. Zirconium dioxide serves as contrast medium. The liquid consists of calcium chloride in aqueous solution with an admixture of polycarboxylate. The powder is mixed with the liquid in a capsule in the triturator for 30 seconds. Once mixed, Biodentine ${ }^{\mathrm{TM}}$ sets in about 12 minutes. During the setting of the cement calcium hydroxide is formed. The consistency of Biodentine TM reminds of that of phosphate cement. ${ }^{3}$

In summary, Biodentine ${ }^{\mathrm{TM}}$ is both a dentin substitute base and a cement for maintaining pulp vitality and stimulating hard tissue formation, i.e. the formation of reactive or reparative (tertiary) dentin. The case report illustrates the use of Biodentine ${ }^{\mathrm{TM}}$ for direct pulp capping.4 


\section{CASE REPORT}

CASE REPORT: A 22 Year old male patient reported to the Department of Conservative Dentistry and Endodontics, Rajarajeshwari Dental College and Hospital, Bangalore, Karnataka, India. With the chief complaint of decay in the lower left back tooth region. In the initial examination revealed a deep carious lesion. The teeth were tested positive on pulp vitality and negative on percussion. Radiographic evaluation showed a deep carious lesion involving enamel, dentin and approximating the pulp. After thorough information to the patient about the treatment consent was obtained block with lignocaine $(2 \%)$ was given followed by rubber dam application. Following cavity preparation the carious dentin was completely excavated with slow speed hand piece and spoon excavator. In the process the pulp cavity was exposed iatrogenically. Clinically the pulp tissue was vital without any major bleeding, so that maintenance of tooth vitality by direct pulp capping was decided upon. $\mathrm{NaOCl}$ $(2.5 \%)$ was applied for hemostasis, clearing and disinfecting the cavity. Biodentine ${ }^{\mathrm{TM}}$ was chosen for direct pulp capping. Mixed as recommended by the manufacturer, and was applied on the exposed pulp tissue as direct capping. About twelve minutes after mixing, when the material had set the cavity was restored with composite. The outcome of the performed treatment was evaluated by pulp vitality tests and Radiographic evaluation for any periapical changes at 1month, 3month and 6 months interval. At the end of 6-month follow up: Clinically teeth were asymptomatic. The teeth responded positive for pulp vitality and negative for percussion tests. The radiographic evaluation showed no periapical changes.

DISCUSSION: The primary aim of pulp capping material is to induce a specific hard tissue formation by pulp cells that seal the exposure site and ultimately contribute to continued pulp vitality. It is known that calcium silicate cements have the ability to release calcium and hydroxyl ions and to form hydroxyapitite crystals on the surface after contact with phosphate containing liquids like body fluids. It can be assumed that Biodentine exhibits the same effects as calcium hydroxide, Due to the release of calcium and hydroxyl ions, when in contact with water. 5 Hence, Biodentine is biocompatible it does not damage pulpal cells, and is capable of stimulating tertiary dentin formation. Compared to other materials such as Mineral Trioxide Aggregate, Biodentine is less technique sensitive ${ }^{5}$. Setting time of biodentine is $12-15$ mins, compared to MTA which has a setting time $>24$ hrs. Unlike other Portland cement-based products, it is sufficiently stable to be used for pulp protection. Used for pulp capping, the material offers some benefits versus calcium hydroxide: It is mechanically stronger, less soluble and produces tighter seal. This qualifies it for avoiding three major drawbacks of calcium hydroxide, Material resorption, Mechanical instability and failure of preventing microleakage. Replacement of the dentin layer and enamel layer with a composite restoration was performed during the same appointment. As it is important to seal the cavity against bacterial invasion in a one--stage procedure ${ }^{6}$. There is extensive evidence documenting that composite fillings are leak proof, few pertinent data are available for biodentin yet.

CONCLUSION: Biodentine ${ }^{\mathrm{TM}}$ is an interesting product, with the potential of making a major contribution to maintaining pulp vitality in patients judiciously selected for direct pulp capping. 


\section{CASE REPORT}

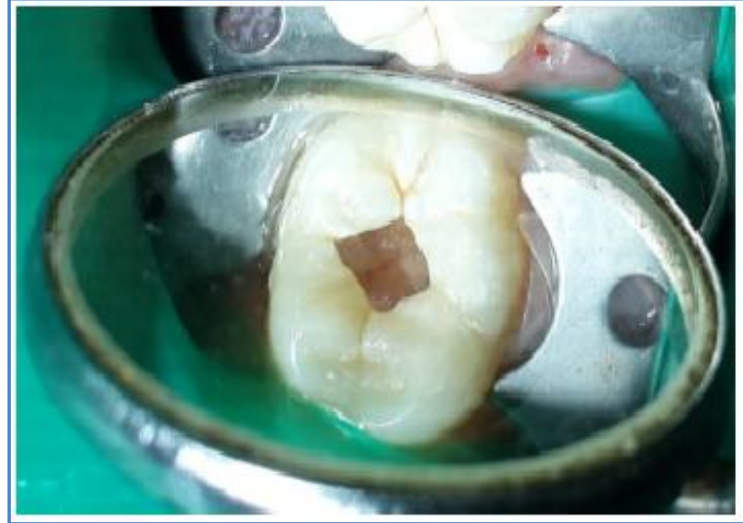

Fig. 1: Pre-operative picture showing deep carious lesion

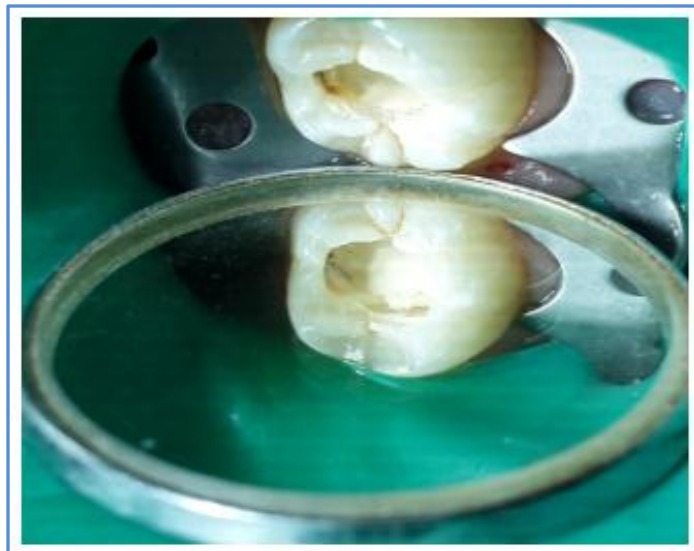

Fig. 3: Placement of biodentine over the exposure site

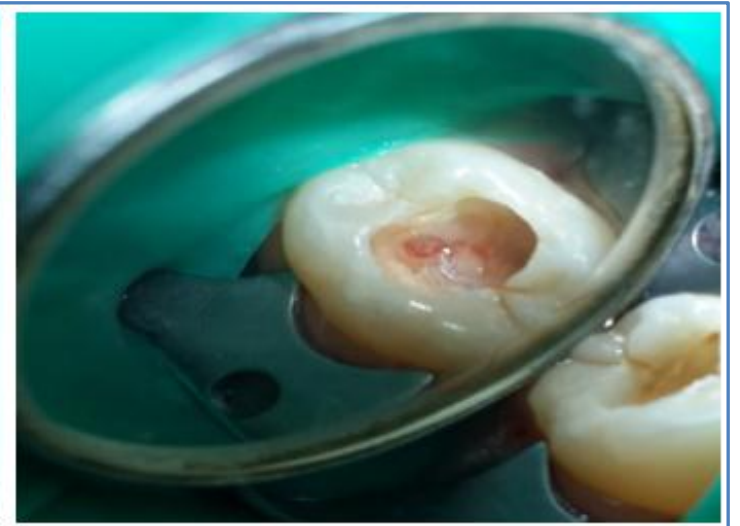

Fig. 2: Iatrogenic exposure of pulp

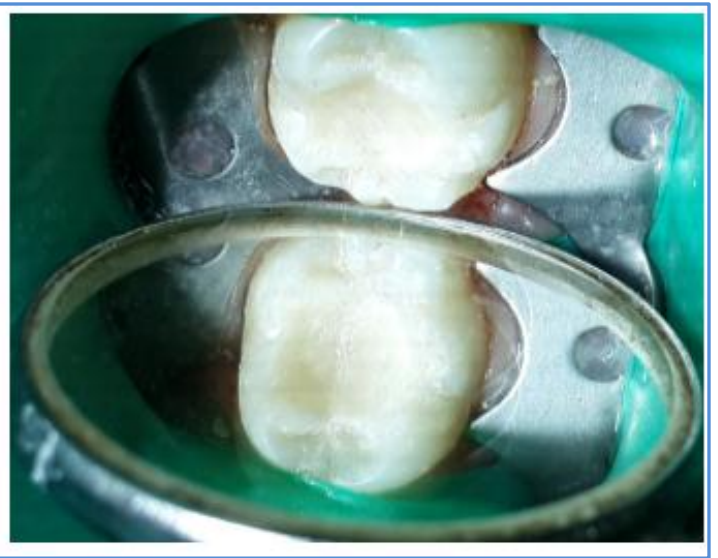

Fig. 4: Final restoration with composite

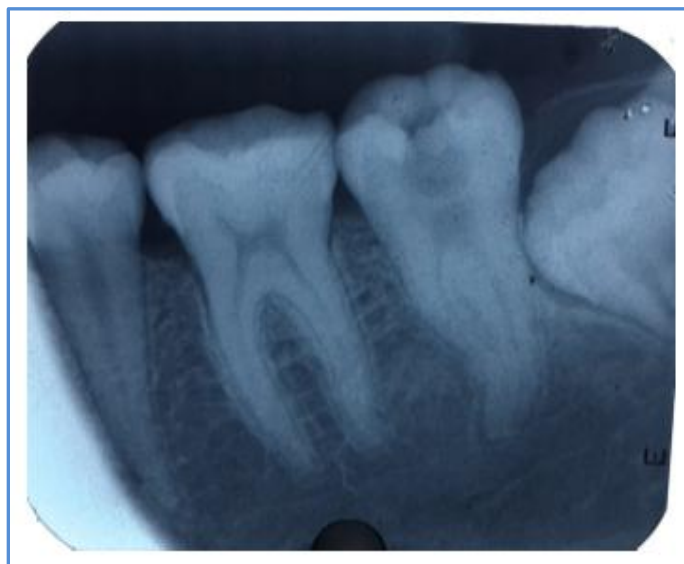

Fig. 5: Preoperative radiograph

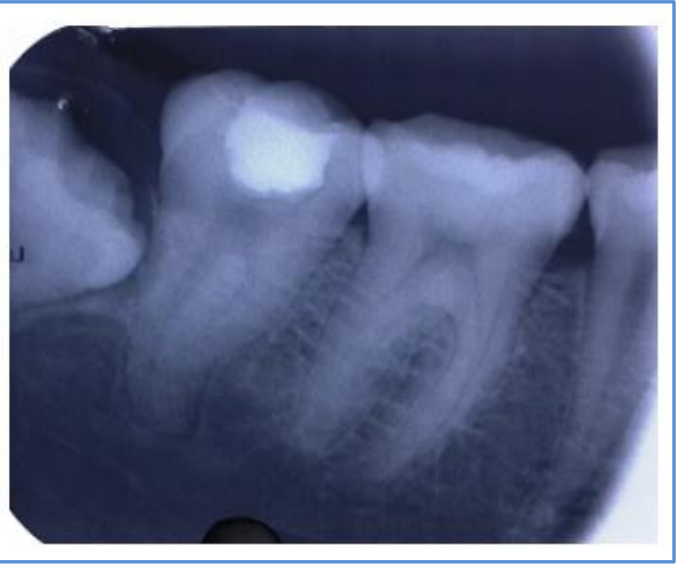

Fig. 6: Evaluation after 1 month 


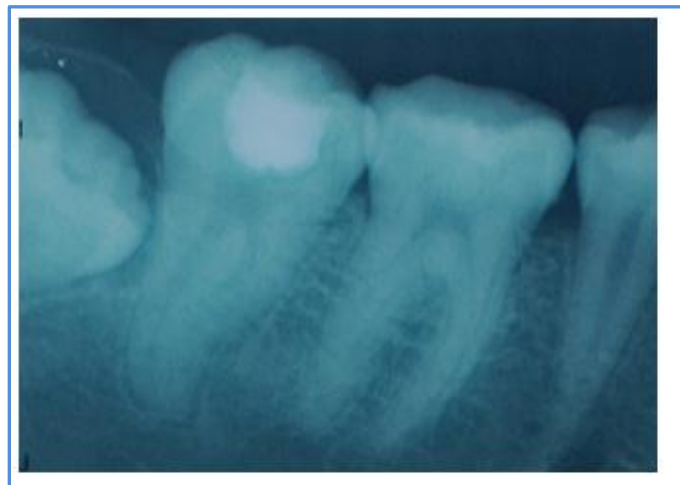

Fig. 7: Evaluation after 3 months

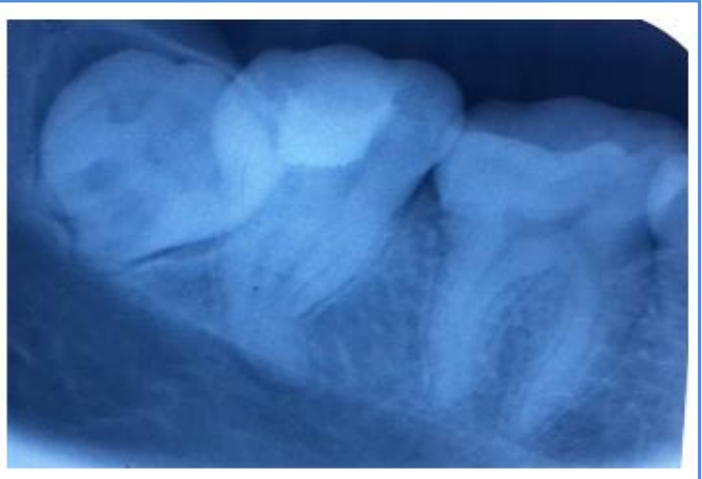

Fig. 8: Evaluation after 6 months

\section{REFERENCES:}

1. Alicja Nowicka, Katarzyna Sporniak-Tutak, Damian Lichota et al. Response of human dental pulp capped with Biodentine and Mineral Trioxide Aggregate. J ENDOD 2013; 39(6): 743-47.

2. Gilles Koubi, Pierre Colon, Jean -Claude Franquin et al. Clinical evaluation of the performance and safety of a new dentin substitute, Biodentine, in the restoration of posterior teeth - a prospective study. Clin oral invest 2014.

3. Harpreet Singh, Mandeep Kaur, Sheenam Markan, Pooja Kapoor. Biodentine: A promising Dentin Substitute. J Interdiscipl Med Dent Sci 2014; 2 (5).

4. Saravanapriyan Soundappan, Jothi Latha Sundaramurthy, Sandhya Raghu, Velmurugan Natanasabapthy et al. Biodentine versus Mineral Trioxide Aggregate versus Intermediate Restorative Material for Retrograde Root End Filling: An In Vitro Study. J Dent 2014 11(2): 143-49.

5. Priyalakshmi.S, Manish R. Review on Biodentine- A Bioactive Dentin Substitute. IOSR-JDMS 2014; 13(1): 13-17.

6. Grech L, Mallia B, Camilleri. Characterization of set Intermediate Restorative Material, Biodentine, Bioaggregate and a Prototype calcium silicate cement for use as root end filling materials. Int Endod J 2013; 46: 632-41. 


\section{CASE REPORT}

\section{AUTHORS:}

1. Nitish Kumar Neelagiri

2. Geeta I. B.

3. Shubhashini Narayanaswamy

4. Vinay Chandra R.

5. Aniketh T. N.

\section{PARTICULARS OF CONTRIBUTORS:}

1. Post Graduate, Department of Conservative Dentistry \& Endodontics, Rajarajeswari Dental College \& Hospital, Bangalore.

2. Professor \& HOD, Department of Conservative Dentistry \& Endodontics, Rajarajeswari Dental College \& Hospital, Bangalore.

3. Reader, Department of Conservative Dentistry \& Endodontics, Rajarajeswari Dental College \& Hospital, Bangalore.

\section{FINANCIAL OR OTHER} COMPETING INTERESTS: None
4. Professor, Department of Conservative Dentistry \& Endodontics, Rajarajeswari Dental College \& Hospital, Bangalore.

5. Post Graduate, Department of Conservative Dentistry \& Endodontics, Rajarajeswari Dental College \& Hospital, Bangalore.

\section{NAME ADDRESS EMAIL ID OF THE CORRESPONDING AUTHOR:}

Dr. Nitish Kumar Neelagiri,

Department of Conservative Dentistry \&

Endodontics, Rajarajeswari Dental College \&

Hospital, Bangalore.

E-mail: niteshkumar0131@gmail.com

Date of Submission: 03/04/2015.

Date of Peer Review: 04/04/2015.

Date of Acceptance: 17/04/2015.

Date of Publishing: 27/04/2015. 